following analytes: IL-6, IL-12, IL-17, IL-23, IL-27, TNF $\alpha$, IFN $\gamma, \mathrm{MIF}, \mathrm{OPG}$, SOST, GM-CSF, VEGF, DKK-1, S100A8, MMP-3 and CRP. To assess pain levels, patients indicated on a scale of $0-10$ the amount of back pain experienced at night and separately the amount of back pain experienced at any time during the last week. They also completed a Bath Ankylosing Spondylitis Disease activity Index (BASDAI) questionnaire to assess disease activity. Cytokine concentrations in patients with ERA were compared with those of controls. The levels were also correlated with the two measures of back pain as well as individual questions from the BASDAI questionnaire.

Results: Of all 54 samples tested, 11 patients and 5 controls crossreacted with the negative control for the assay and thus were excluded from analysis. Based on MRI scan results, 14 patients had ERA, 8 had biomechanical pain, 7 had other subtypes of JIA, and 2 had non-specific features of spinal inflammation. The median overall back pain and the total BASDAI scores for the ERA group were 2.4 (IQR=1.45-5.55) and $2.5(\mathrm{IQR}=0.75-6.25)$ respectively, suggesting well controlled disease on treatment and minimal residual symptoms. There was no statistical difference between cytokine levels in the ERA group compared to controls when corrected for multiple testing, with the exception of IL-12 which was significantly higher in controls $(p=0.003)$. No correlation was found between cytokines and pain scores (at night or overall during the last week) or with the overall BASDAI score or any of the sub-component questions of the BASDAI questionnaire.

Conclusion: Well-controlled ERA patients on treatment have similar cytokine profiles as healthy controls and they do not correlate with clinical pain scores or disease activity.

Disclosure of Interests: None declared

DOI: 10.1136/annrheumdis-2019-eular.2827

\section{AB0230B STUDY OF CELIAC DISEASE ANTIBODIES IN PATIENTS WITH JUVENILE ONSET RHEUMATOLOGICAL DISORDERS}

Eman Hassan ${ }^{1,2}$, Nermen Magdy ${ }^{3,4} .{ }^{1}$ Egypt, Internal Medicine Rheumatology, Alexandria, Egypt, ${ }^{2}$ Faculty of Medicine, University of Alexandria, Internal Medicine Rheumatology, Alexandria, Egypt, ${ }^{1}$ Egypt, Internal Medicine Rheumatology, Alexandria, Egypt, ${ }^{4}$ Faculty of Medicine, Internal Medicine Rheumatology, Alexandria, Egypt

Background: Preclinical autoimmunity can be detected in the form of circulating autoantibodies in the peripheral blood many years preceding the onset of the clinical disease. Celiac disease (CD) is an immunological response to gluten in genetically susceptible people. The clinical presentation of CD includes abdominal pain, diarrhea and nutritional deficiencies. However; clinical symptoms could be misleading in most of the patients presenting subclinical forms with only minor gastroenterological symptoms. Celiac disease will present in older children by atypical presentation. Most celiac disease patients show atypical symptoms and may remain undiagnosed; which makes screening is mandatory in high-risk patients with autoimmune diseases. This collectively highlights the need to check for clinical evidence of celiac disease among patients diagnosed as juvenile onset rheumatic diseases

Objectives: The aim of the work is to screen for celiac disease antibodies (anti-tissue transglutaminase $\lg A$ and $\lg G$ auto-antibodies) in the serum of juvenile onset rheumatic diseases patients in comparison with normal subjects

Methods: Serum tTG (both $\lg A$ and $\lg$ ) level was detected in 60 juvenile onset rheumatic patients and 20 age and sex matched healthy controls. We also assessed different clinical and laboratory markers of disease parameters; 31 juvenile onset systemic lupus erythematosus (SLE), 21 juvenile onset idiopathic arthritis (JIA), 4 juvenile onset systemic sclerosis and 2 juvenile onset behcet's disease and each disease activity score. We also correlated serum transglutaminase auto-antibodies with each disease activity. Endoscopic examination and histopathologic examination, for the patients with a least one positive anti-tTG Ab was done

Results: Serum anti-tTG Abs was no statistical significant differences in the patients group than in the control group, and there was no correlation between anti-tTG Abs with positive disease activity score

Conclusion: The presence of concomitant $C D$ and another rheumatic disorder in the same patient is unlikely

\section{REFERENCES:}

[1] Roth B. Transglutaminase and peptidylarginine deiminase in the pathogenesis of autoimmune diseases. Doctorial Dissertation series. Sweden, Lund: Media-Tryck 2008
[2] Vojdani A. Antibodies as predictors of complex autoimmune diseases and cancer. International Journal of Immunopathology and Pharmacology. 2008; 21(3):553-566.

Disclosure of Interests: None declared

DOI: 10.1136/annrheumdis-2019-eular.848

\section{Rheumatoid arthritis - prognosis, predictors and outcome}

\section{AB0231 PAIN THRESHOLD IN RHEUMATOID ARTHRITIS AND ITS RELATED FACTOR}

Aysegul Altun Guvenir, Aslı Calıskan Uckun, Fatmagul Yurdakul, Hatice Bodur. Ankara N Hatice Bodur umune Training and Research Hospital, Physical Medicine and Rehabilitation, ANKARA, Turkey

Background: Pain remains the most important challenge for rheumatoid arthritis (RA) patients. In order to cope with pain in RA, clinicians firstly need to understand the degree of patients'pain. When self reported measures such as visual analog scale were used, inconsistency between clinicians' and patients' ratings of pain was demonstrated (1). As a result inability to fully assess the pain will cause the inability to fully assess the impact of pain on disease severity. Measuring pressure pain threshold with pressure algometry may provide additional advantages for the evaluation of pain and disease severity when compared with self-reported measures.

Objectives: The aim of this study was to evaluate the pain threshold and to determine the variables associated with pain threshold in the patients with RA.

Methods: The current study included 100 RA patients and 80 age-sex matched controls with non-inflammatory chronic low back pain. Clinica parameters, functional status, disease activity, pain, fatigue, depression, anxiety, pain catastrophizing and laboratory activity of RA patients were recorded. The pressure pain thresholds on the dominant thumb nail bed, trapezius and wrist of the two groups were measured by the algometer. The pain thresholds of the paients with RA and controls were compared. Moreover, the relation between pain threholds and all evaluated parameters in RA patients was analysed.

Results: The mean age of RA patients was $55.93 \pm 10.81$; the mean age of the controls was

$54,50 \pm 9,47$. The pain thresholds of RA patients and controls were statistically similar in all areas. When the parameters found to be significantly correlated with pain threshold in RA patients were analyzed by regression analysis, depression was the only factor associated with low pain threshold in all areas (Table 1).

Conclusion: We found that depression was the only factor associated with low pain threshold in the patients with RA. The use of pressure algometry in the evaluation of chronic pain could offer an additional method to detect pain/depression overlap.

Table 1. The correlation between pain threshold values and demographic, clinical and laboratory parameters of RA patients

\begin{tabular}{lcccccc}
\hline & \multicolumn{2}{c}{ Nail PT } & \multicolumn{2}{c}{ Wrist PT } & \multicolumn{2}{c}{ Trapezius PT } \\
\hline & $\mathrm{p}$ & $\mathrm{r}$ & $\mathrm{p}$ & $\mathrm{r}$ & $\mathrm{p}$ & $\mathrm{r}$ \\
\hline Age & 0,551 & 0,060 & 0,705 & 0,038 & 0,806 & 0,025 \\
BMI (kg/m ${ }^{2}$ ) & 0,174 & $-0,137$ & 0,066 & $-0,185$ & $0,046^{*}$ & $-0,200$ \\
$\begin{array}{l}\text { Duration of disease } \\
\text { (year) }\end{array}$ & 0,247 & $-0,117$ & 0,144 & $-0,147$ & 0,364 & 0,092 \\
Morning stiffness & & & & & & \\
(minute) & 0,415 & $-0,082$ & 0,592 & $-0,054$ & 0,542 & $-0,062$ \\
Tender joint counts & & & & & & \\
Swollen joint counts & $0,046^{*}$ & $-0,200$ & 0,099 & $-0,166$ & 0,361 & $-0,092$ \\
CRP & 0,109 & $-0,161$ & 0,227 & $-0,122$ & 0,522 & $-0,065$ \\
ESR & 0,681 & $-0,042$ & 0,144 & 0,147 & 0,076 & 0,178 \\
VAS & 0,952 & $-0,006$ & 0,330 & 0,098 & 0,453 & 0,076 \\
PCS & 0,056 & $-0,192$ & 0,069 & $-0,182$ & 0,086 & $-0,173$ \\
Fatigue Intensity Scale & $0,013^{*}$ & $-0,248$ & $0,013^{*}$ & $-0,249$ & $0,034^{*}$ & $-0,212$ \\
HADS-Anxiety & 0,107 & $-0,162$ & 0,145 & $-0,147$ & 0,422 & $-0,081$ \\
HADS-Depression & 0,157 & $-0,143$ & 0,118 & $-0,157$ & 0,285 & $-0,108$ \\
HADS-Total & $0,010^{*}$ & $-0,256$ & $0,005^{*}$ & $-0,282$ & $0,003^{*}$ & $-0,293$ \\
HAQ & $0,026^{*}$ & $-0,223$ & $0,014^{*}$ & $-0,245$ & $0,024^{*}$ & $-0,225$ \\
DAS28-ESR & $<0,001^{*}$ & $-0,264$ & $0,000^{*}$ & $-0,287$ & $<0,001^{*}$ & $-0,315$ \\
DAS28-CRP & $0,013^{*}$ & $-0,248$ & 0,091 & $-0,170$ & 0,211 & $-0,126$ \\
\hline
\end{tabular}

PT: pain threshold, BMI: Body mass index, PCS: Pain Catastrophizing Scale, VAS: Visual Analog Scale, HAQ: The health assessment questionnaire ${ }^{*} p<0.05$ 


\section{REFERENCE:}

[1] Bagnato G, De Andres I, Sorbara S, Verduci E, Corallo G, Ferrera A, et al. Pain threshold and intensity in rheumatic patients: correlations with the Hamilton Depression Rating scale. Clinical rheumatology. 2015;34(3):55561

Disclosure of Interests: None declared

DOI: 10.1136/annrheumdis-2019-eular.7390

\section{AB0232 TIGHT CONTROL MANAGEMENT OF EARLY RA ACHIEVES MORE PATIENTS IN REMISSION AT 6 MONTHS AND IMPROVES PAIN, FUNCTION AND FATIGUE AT 24 MONTHS COMPARED TO A CONTROL GROUP}

Maria Aronsson ${ }^{1,2}$, Annika Teleman ${ }^{1}$, Stefan Bergman ${ }^{2,3}$, Kristina Forslind $^{4}$, Maria Andersson ${ }^{2,5}$. Background: Even with modern antirheumatic treatment, many patients experience chronic pain and loss of function despite less disease activity. The understanding of how this chronic pain development can be prevented is sparse. There is furthermore little knowledge if a tight control management without specific treatment schemes and instructions for the physicians to follow could increase the remission frequency.

Objectives: To determine whether solely a tight control management of patients, without further intervention in the physician's work, can increase the remission frequency and improve patient reported outcome in early RA.

Methods: In all, 96 patients with early RA were consecutively included in a tight control study group (TCS). Inclusion criteria were early RA patients ( $<13$ month duration) with no previous history of rheumatic disease. A control group, with so far 27 patients receiving standard care, has been analyzed. The tight control management included monthly visits to the physician the first 6 months, followed by visits at 9,12 and 24 months. If not in remission at 6 months the patients' monthly visits could continue to 12 months. The study did not include any treatment guidelines for the physicians. Pain, Fatigue and the Patient's Global Assessment of the General Health (PtGA) were reported on a Visual Analogue Scale (VAS). The 28 tender and swollen joint score, DAS28, C-Reactive protein, Erythrocyte sedimentation rate and Health Assessment Questionnaire (HAQ) were assessed.

Results: There was no significant difference between the groups at baseline in gender, age, smoking, disease duration, disease activity, pain, $H A Q$, fatigue, RF and ACPA. There was a significant difference between groups in outcome, with $70 \%$ of TCS and $37 \%$ of control patients in remission (DAS28 <2.6), $p=0.010$, at 6 months, figure 1 A. At 12 and 24 months there was no significant difference in the remission frequency, figure $1 \mathrm{~A}$. The TCS group improved more than the control group in Pain, Fatigue and $H A Q$ during follow up, figure $1 B-D$. The median VAS pain at 24 months was 11 (IQR 2-22) in the TCS group and 22 (IQR 6-42) in the control group, $p=0.036$. The median VAS fatigue was 13 (IQR 238 ) in the TCS group and 33 (IQR 8-70) in the control group $\mathrm{p}=0.028$ at 24 months. The difference in $\mathrm{HAQ}$ reached significant numbers solely at 6 months where the median was 0.13 (IQR $0-0.50$ ) in the TCS group and 0.57 in the control group (IQR 0.16-0.94).

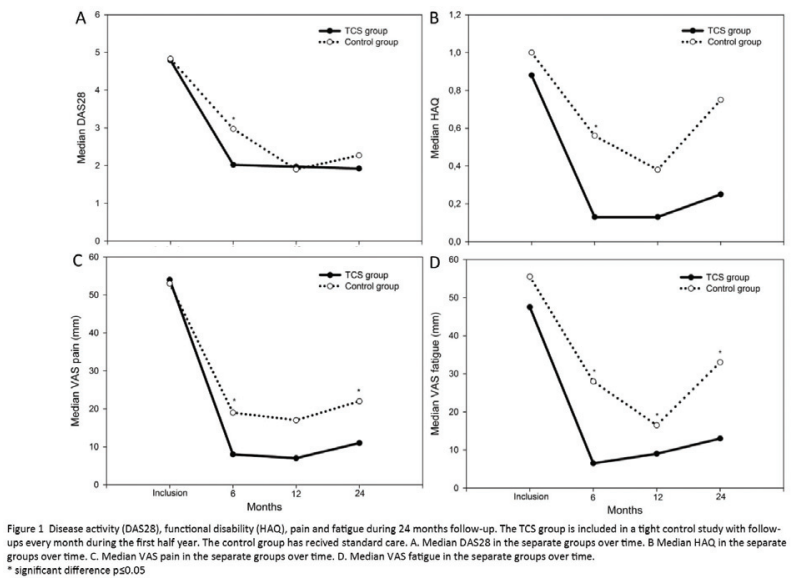

Figure 1
Conclusion: Patients in tight control reached remission faster than patients receiving standard care. They also reported less pain and fatigue, despite the fact that there was no difference in remission frequency at 24 months. The results indicate that a tight control scheme could influence pain development in patients with early RA, but further studies are required.

Disclosure of Interests: Maria Aronsson: None declared, Annika Teleman Consultant for: Pfizer expert Group 2016, Sanofi expert Group 2017. No association with this study., Stefan Bergman: None declared, Kristina Forslind: None declared, Maria Andersson: None declared DOI: 10.1136/annrheumdis-2019-eular.6938

\section{$\mathrm{AB} 0233$ \\ ASSESSMENT OF THE RESPONSE TO THERAPY WITH CSDMARDS AND BDMARDS IN PATIENTS WITH RHEUMATOID ARTHRITIS}

Vladimira Boyadzhieva, Nikolay Stoilov. University Hospital "St Ivan Rilski", Rheumatology, Sofia, Bulgaria

Background: The significant economic burden of rheumatoid arthritis (RA) and new treatment options, consuming much of the healthcare resources, pay attention on appropriate management strategies. These strategies are due to the need for an adequate assessment of the response to treatment and changes if it is necessary. Currently used American College of Rheumatology (ACR) response criteria and disease activity score (DAS28CRP) allows on time evaluation of the results and facilitate decision proc ess when is the right time to switch patient's therapy.

Objectives: The aim of the study is to evaluate the effectiveness and response to the treatment with csDMARDS and/or corticosteroid and bDMARDS in patients with RA after one year follow up period using ACR 20/50/70/90 response criteria and DAS28-CRP.

Methods: A total of 220 patients with a mean age $55.05 \pm 10.63$ SD meeting the 1987 ACR classification criteria for RA were included in the study. The mean disease duration was $9.97 \pm 5.78 S D$. Patients were stratified according to treatment regimens into age-matched treatment groups 96 on csDMARDs and/or corticosteroid (Sulfasalazine ? 15, Methylprednisolone ? 15, Chloroquine - 16, Methotrexate - 20, Methotrexate and Methylprednisolone -30) and 124 on bDMARD (Tocilizumab ? 30, Certolizumab pegol ? 16, Golimumab - 22, Etanercept - 20, Adalimumab 20. Rituximab - 16). All participants were evaluated for ACR 20/50/70/90 response to therapy on 6-th and 12th month of the follow up period, DAS28-CRP was calculated via licensed calculator. Comparison was performed by analysis variance ANOVA

Results: The majority of patients (71\%) on bDMARDS achieved ACR 50 response criteria and $16 \%$ achieved ACR 70 , indicating a significantly better therapeutic effect compared to the group on csDMARDS (ACR 50 - $11 \%$ ACR $70-1 \%$ ) at 6 th month follow up ( $p<0.001, p=0.002)$. Only $3 \%$ of bDMARDS patients achieved ACR 90 response on the $6^{\text {th }}$ month. Most of the patients on csDMARDs (64\%) reach ACR 20 response criteria after the same period of therapy. At 6th month of follow-up, best response was achieved by the patients on combined MTX and corticosteroid (CS) therapy (50\%) achieved ACR 20, 27\% - ACR 50 and $3 \%$ ACR 70. At the 12th month, a significant proportion of patients on bDMARDS reached the ACR 50 (36\%) and ACR $70(52 \%)$ response criteria compared to the csDMARDS group(ACR 50 - 20\%, ACR $70-$ $10 \%)(p=0.0102 ; p<0.001)$. We evaluated the response to therapy according to the DAS28-CRP categories. On baseline patients on bDMARDS had higher disease activity in comparison to patients on csDMARDS $(p=0.0004)$. In the follow-up to the 6th month, we observed a significant reduction in the proportion of patients (on bDMARDS) with severe disease activity - $2.4 \%$, compared to csDMARDS group - $13.5 \%$ ( $p=0.0019$ ). In $26.6 \%$ of patients on bDMARDS, we found minimal dis ease activity, which was significantly more than $13.5 \%$ for the csDMARDS group ( $p=0.0186$ ). A remission was found in $5.6 \%$ of patients on bDMARDS which is significantly less than $14.6 \%$ for the group on csDMARDS. In contrast to the results obtained at the 6th month of follow-up, at 12th month we observed significantly higher percentage of patients on bDMARDS who had reached the remission category - 29.8\%, in comparison with csDMARDS group - 12.5\%, ( $p=$ 0.0025).

Conclusion: Patient treated with bDMARDs showed better response to treatment and lower disease activity than those on therapy with csDMARDs within a period of 12 months of treatment. Current management strategies should focus on switching on another medication earlier in the treatment plan when patients inadequately respond to therapy after 6 months of follow up.

Disclosure of Interests: None declared 